\title{
Monitoring of the optical spectrum of comet 2I/Borisov at the VLT
}

\author{
Emmanuel Jehin ${ }^{1}$, Yang Bin ${ }^{2}$, Olivier Hainaut ${ }^{3}$, Cyrielle Opitom ${ }^{2,4}$, Jean Manfroid ${ }^{1}$, Youssef \\ Moulane $^{2}$, Alan Fitzsimmons ${ }^{5}$, Colin Snodgrass ${ }^{4}$, and Karen Meech ${ }^{6}$ \\ ${ }^{1}$ Université de Liège (ULiege), STAR Institute, Liège, Belgium (ejehin@uliege.be) \\ ${ }^{2}$ European Southern Observatory (ESO), Santiago, Chile \\ ${ }^{3}$ European Southern Observatory (ESO), Garching, Germany \\ ${ }^{4}$ Institute for Astronomy, University of Edinburgh, Royal Observatory, UK \\ ${ }^{5}$ Astrophysics Research Centre, Queen's University Belfast, UK \\ ${ }^{6}$ Institute for Astronomy, Honolulu, USA
}

2I/Borisov is the first active interstellar comet observed in the Solar Sytem, allowing for the first time to sample the composition of a planetary building block from an extrasolar system. We report on the monitoring of 2I with the FORS low resolution spectrograph of the ESO VLT at Paranal during four months, from November 19, 2019 to March 20, 2020. We collected a dozen spectra at 8 different epochs allowing to follow the evolution of the comet activity and composition around perihelion. We also observed with the same instrumental setup an Oort Cloud comet, C/2019 U6 (Lemmon), at about the same heliocentric and geocentric distance than 2I/Borisov at perihelion ( $r h=$ Delta=2 au) and with similar AfRho value and $Q(C N)$ in order to use it as a reference for the Solar System. The usual species are detected in the optical spectrum of 2I (CN, C3, C2, and NH2) and their production rates and abundance ratios are computed. The dust production rate and colors are also derived, compared to C/2019 U6 and other comets of the Solar System, and their evolutions are followed with the heliocentric distance. 
A/2019 U6 (Feb, 28, 2020) vs 2I/Borisov (Dec. 5, 2019)



Fig1. Comparison of the optical spectra of the interstellar comet 2I/Borisov at perihelion ( $r h=$ Delta =2,0 au) and the Oort Could comet C/2019 U6 (Lemmon) observed with FORS at the ESO VLT (Paranal Observatory). C/2019/U6 was chosen for comparison as a Solar System comet with a typical composition. It was also at the about the same distances to the Sun and Earth, and it had about the same dust and $\mathrm{CN}$ production rates as 2I/Borisov as measured by the TRAPPIST-South telescope. 